This is the post peer-review accepted manuscript of:

C. Orozco, A. Borghetti, S. Lilla, G. Pulazza and F. Tossani, "Comparison Between Multistage Stochastic Optimization Programming and Monte Carlo Simulations for the Operation of Local Energy Systems," 2018 IEEE International Conference on Environment and Electrical Engineering and 2018 IEEE Industrial and Commercial Power Systems Europe (EEEIC / I\&CPS Europe), Palermo, 2018, pp. 1-6.

The published version is available online at:

https://doi.org/10.1109/EEEIC.2018.8494563

(C) 2018 IEEE. Personal use of this material is permitted. Permission from IEEE must be obtained for all other uses, in any current or future media, including reprinting/republishing this material for advertising or promotional purposes, creating new collective works, for resale or redistribution to servers or lists, or reuse of any copyrighted component of this work in other works. 


\title{
Comparison Between Multistage Stochastic Optimi- zation Programming and Monte Carlo Simulations for the Operation of Local Energy Systems
}

\author{
C. Orozco, A.Borghetti, S. Lilla, G. Pulazza, F. Tossani \\ Department of Electrical, Electronic and Information Engineering \\ University of Bologna, Italy
}

camilo.orozco2@unibo.it, alberto.borghetti@unibo.it, stefano.lilla3@unibo.it, giorgiapulazza@libero.it, fabio.tossani@unibo.it

\begin{abstract}
The paper deals with the day-ahead optimization of the operation of a local energy system consisting of photovoltaic units, energy storage systems and loads aimed at minimizing the electricity procurement cost. The local energy system may refer either to a small industrial site or to a residential neighborhood. Two mixed integer linear programming models are adopted, each for a different representation of the battery: a simple energy balance constraint and the Kinetic Battery Model. The paper describes the generation of the scenarios, the construction of the scenario tree and the intraday decision-making procedure based on the solution of the multistage stochastic programming. Moreover, the daily energy procurement costs calculated by using the stochastic programming approach are compared with those calculated by using the Monte Carlo method. The comparison is repeated for two different sizes of the battery and for two load profiles.
\end{abstract}

Keywords-Energy scheduling; Local energy system; Mixed integer linear programming; Stochastic programming; Scenario reduction; Monte Carlo method; Kinetic Battery Model.

\section{INTRODUCTION}

This paper deals with an electric local energy system with the presence of renewables, such as the power system of an industrial site or of a residential neighborhood. The considered system includes a photovoltaic (PV) unit capable to provide a significant part of the local energy consumption and it is also equipped with an energy storage unit to fully exploit the available renewable energy source even for the case of a limited capability of the external utility network to which the system is connected.

The daily operation of the battery unit is addressed as an optimization problem with a $24 \mathrm{~h}$ horizon, with the aim to minimize the electricity procurement cost. The inputs are the forecasting of the PV production and of the local loads.

Since the forecasts of both PV production and load consumption are affected by significant uncertainties, either stochastic optimization approaches or Monte Carlo simulations are typically adopted to solve this kind of problems (e.g., [1][3]).

In this paper these two different approaches are compared by using a mixed integer linear programming model of the local energy system, having the two following characteristics:

- a 15 minutes time discretization, which appears more suitable

This work has received funding from the European Union's Horizon 2020 research and innovation programme under the Marie Skodowska-Curie grant agreement No 675318 (INCITE) and from the Electronic Components and Systems for European Leadership Joint Undertaking under grant agreement No 737434 (CONNECT). for the energy management of the local system than the usual 1-hour time step;

- the use of the kinetic battery model (KiBaM) [4]-[6] (also adopted in the Homer Energy software) for the representation of the battery state of charge, which is more detailed than the simple energy balance one (other detailed models have been proposed in e.g. [7]).

In order to better adapt the day-ahead solution to the actual intraday operating conditions, the stochastic optimization problem is formulated as a multistage decision problem in which the battery output set points are decided at the beginning of the day and subsequently other three times during the day (every 6 hours). The five-stage stochastic optimization problem (where the decisions are taken at the beginning of each stage) needs a scenario tree model that is built by using the $k$-means clustering method.

The structure of the paper is the following. Section II describes the linear programming model of the local energy system. Section III describes the multistage stochastic programming (SP) procedure with the scenario generation and the construction of the scenario tree. Section IV illustrates the test results and the comparison between the daily energy procurement costs calculated by using the five-stage stochastic optimization with those calculated by using the Monte Carlo solution (i.e., the average of the optimal decisions provided by the deterministic solution of each of the scenarios used for the construction of the tree). Section V concludes the paper.

\section{MODEL OF THE LOCAL ENERGY SYSTEM}

We focus here on the solution of the day-ahead scheduling, which is in general associated with a real time control of the integrated PV-storage system, as dealt with in e.g., [8],[9].

A typical aim of the energy management system is the minimization of the production costs associate with PV, storage units and the power exchange with the external network to feed the internal load in a time horizon $T$ :

$$
O F=\sum_{t \in T}\left(p_{t}^{i m p} P_{t}^{i m p}-p_{t}^{\exp } P_{t}^{\exp }\right) \Delta t
$$

where:

- parameters $p_{t}^{i m p}$ and $p_{t}^{\text {exp }}$ are the prices (in $€ / \mathrm{kWh}$ ) of the energy exchanged with the external grid (bought and sell, respectively); 
- nonnegative variables $P_{t}^{\text {imp }}$ and $P_{t}^{\text {exp }}$ are the values of the power absorbed and injected into the external grid (in $\mathrm{kW}$ );

- parameter $\Delta t$ is the 15-minutes time step (in h).

A. Model with a simple representation of the battery state of charge

The constraints of the model, to be described next, are the following for all the time intervals $t$.

$$
\begin{gathered}
P_{t}^{p v}-P_{t}^{b}+P_{t}^{\text {grid }}-P_{t}^{\text {load }}-L_{t}=0 \\
P_{t}^{b}=P_{t}^{c}-P_{t}^{d} \\
P_{t}^{c} \leq\left(1-u_{t}^{b}\right) M^{b} \text { and } P_{t}^{d} \leq u_{t}^{b} M^{b} \\
-P_{r}^{b} \leq P_{t}^{b} \leq P_{r}^{b} \\
E_{t}^{b}=E_{\max } S O C_{t} \\
S O C_{t}=S O C_{t-1}+P_{t}^{b} \Delta t / E_{\max } \forall t>0 \\
S O C_{t=0}=S O C_{0}, S O C_{t=T \mid}=S O C_{\text {end }} \\
S O C_{\min } \leq S O C_{t} \leq S O C_{\max } \\
L_{t}=L_{t}^{d}+L_{t}^{c} \\
L_{t}^{c}=\frac{1-\eta_{c}}{\eta_{c}} P_{t}^{c} \text { and } L_{t}^{d}=\left(1-\eta_{d}\right) P_{t}^{d} \\
P_{t}^{\text {grid }}=P_{t}^{\text {imp }}-P_{t}^{\exp } \\
P_{t}^{\text {imp }} \leq\left(1-u_{t}^{\text {grid }}\right) M^{\text {grid }} \text { and } P_{t}^{\text {exp }} \leq u_{t}^{\text {grid }} M^{\text {grid }} \\
-P_{r}^{\text {grid }} \leq P_{t}^{\text {grid }} \leq P_{r}^{\text {grid }}
\end{gathered}
$$

where $P_{t}^{p v}$ is the active power injected into the system by the PV unit, $P_{t}^{\text {load }}$ is the power adsorbed by the internal loads; $P_{t}^{b}$ is the battery power output (nonnegative $P_{t}^{c}$ and $P_{t}^{d}$ are the battery power outputs during charges and discharges, respectively); $P_{\text {grid }}^{t}$ is the power exchanged with the external network (nonnegative $P_{t}^{i m p}$ and $P_{t}^{\exp }$ are the imported power and exported power, respectively), $L_{t}$ are the losses of the battery converter ( $L_{t}^{c}$ and $L_{t}^{d}$ are losses during charges and discharges); $E_{t}^{b}$ is the energy level in the battery; $S O C_{t}$ is the battery state of charge.

The definition of the parameters and, in parenthesis, the corresponding values adopted in the numerical tests are: $P_{r}^{b}$ is the rated value of the battery output $(630 \mathrm{~kW}), E_{\max }$ is the battery capacity $(630 \mathrm{kWh}) ; \eta_{c}$ and $\eta_{d}$ are the converter efficiency factors for charges and discharges (0.95 and 0.97, respectively); $P_{r}^{\text {grid }}$ is the maximum power that can be exchanged with the grid $(1.5 \mathrm{MW}), S O C_{\min }$ and $S O C_{\max }$ are the minimum and maximum state of charge $(0.1$ and 1 p.u., respectively), $S O C_{0}$ and $S O C_{\text {end }}$ are the initial value and the required final value of the state of charge (both assumed equal to $1 \mathrm{p} . \mathrm{u}$. in the numerical tests).

The constraints represent: the power equilibrium (2); the power output of the battery with the big-M formulation in order to avoid concurrent charge and discharge $\left(u_{t}^{b}\right.$ is a binary variable and $M^{b}$ is equal to $P_{r}^{b}$ ) (3)-(5); the energy stored and the state of charge of the battery (6)-(9); the losses in the battery converter (10)-(11); the exchange with the external grid (12)-(14), where $u_{t}^{\text {grid }}$ is a binary variable and $M^{\text {grid }}$ is equal to $P_{r}^{\text {grid }}$.

\section{B. Model with the kinetic battery model}

The refined model that include the $\mathrm{KiBaM}$ replaces constraints (7) with the following constraints

$$
\begin{gathered}
E_{t}^{b}=q 1_{t}+q 2_{t} \\
q 1_{t}=q 1_{t-1} e^{-k \cdot \Delta t}+\frac{\left(k c E_{t-1}^{b}+P_{t}^{b}\right)\left(1-e^{-k \cdot \Delta t}\right)}{k}+\frac{k_{q 1} P_{t}^{b}}{k} \\
q 1_{t=0}=c S O C_{0} E_{\max } \\
q 2_{t}=q 2_{t-1} e^{-k \cdot \Delta t}+E_{t-1}^{b}(1-c)\left(1-e^{-k \Delta t}\right)+\frac{k_{q 2} P_{t}^{b}}{k} \\
P_{t}^{c} \leq \frac{1}{\eta_{b}} \frac{k c E_{\max }-k q 1_{t} e^{-k \Delta t}-E_{t}^{b} k c\left(1-e^{-k \Delta t}\right)}{1-e^{-k \Delta t}+k_{q 1}} \\
P_{t}^{c} \leq \frac{1}{\eta_{b}} \frac{\left(1-e^{-a \cdot \Delta t}\right)\left(E_{\max }-E_{t}^{b}\right)}{\Delta t} \\
P_{t}^{d} \leq \eta_{b} \frac{q 1_{t} k e^{-k \Delta t}+E_{t}^{b} k c\left(1-e^{-k \Delta t}\right)}{1-e^{-k \Delta t}+k_{q 1}}
\end{gathered}
$$

where $k_{q 1}=c\left(k \cdot \Delta t-1+e^{-k \Delta t}\right), k_{q 2}=(1-c)\left(k \Delta t-1+e^{-k \Delta t}\right)$

The constraints represent: the definitions of readily available charge $q 1_{t}$ and bound charge $q 2_{t}(15)-(18)$; the battery power outputs limitations during charges and discharges phases (19)-(21).

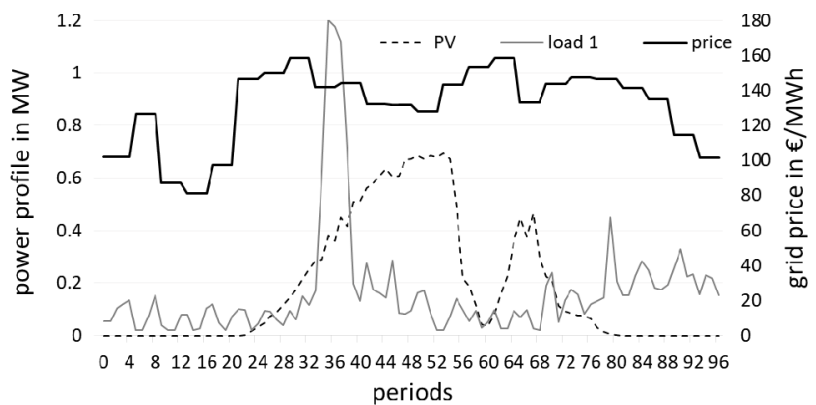

a)

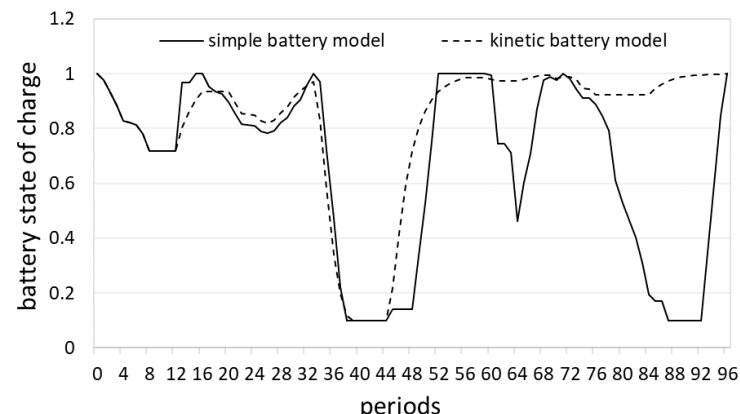

Fig. 1 Deterministic solution: a) Profile of the PV production, load, and grid price; $b$ ) state of charge of the battery calculated by using the simple and kinetic model for profile load 1 . 
The definition of the parameters and, in parenthesis, the corresponding values adopted in the numerical tests are the following: $\eta_{b}$ is the batteries efficiency factor (p.u.) for charges and discharges $(0.9), k$ is the battery rate constant $\left(9.51 \mathrm{~h}^{-1}\right), c$ is the battery capacity ratio $(0.61), a$ is the battery maximum charge rate $(2 \mathrm{~A} / \mathrm{Ah})$.

Fig. 1 shows the results of a deterministic solution of the optimization problem for the considered test system, equipped with a $1 \mathrm{MW}$ PV unit, a storage system of $630 \mathrm{~kW}$ and $630 \mathrm{kWh}$, and a local load with a power consumption of 1.5 MW. Fig. 1 compares the results obtained by using the Kinetic energy model, which uses the parameters value indicated in [6], with those obtained by the simple battery model in which the state of charge is determined by the energy balance. The solution is completed by using Cplex in some tens of milliseconds for both models, with the OF values equal to $€ 36.70$ for the simple model and equal to $€ 61.21$ with the KiBaM.

\section{MULTISTAGE STOCHASTIC OPTIMIZATION PROCEDURE}

We assume that both the load profile and the PV generation are uncertain, whilst, to limit the complexity of the model, prices $p_{t}^{i m p}$ and $p_{t}^{\text {exp }}$ are assumed known.

The decision variable is $P_{t}^{b}$. The decision is taken at the beginning of the day (which is the scheduling horizon) for all the periods of the first 6 hours and they are updated every 6 hours. The 6-hour periods represent the stages following the first one. The values of the other variables are calculated at the end of each stage, for all the periods of the stage.

Denoting the set of scenarios with $\Omega$, the scenario index with $\omega$, and the probability of scenario $\omega$ with $\pi_{\omega}$, the deterministic equivalent of the multistage stochastic problem is the recourse model

$$
\min _{P_{b}^{t}} \sum_{\omega \in \Omega} \pi_{\omega} \cdot O F_{\omega}
$$

with constraints (2)-(14) for the simple battery model and with constraints (2)-(6) and (8)-(21) for the model that includes the KiBaM, other than the nonanticipativity constraints that represent the inability to anticipate the future by forcing the same decisions to be taken for scenarios with the same history.

In the following, we describe the procedures adopted for the generation of set $\Omega$, for construction of the scenario tree that is used in the recourse model, and the intraday decisionmaking procedure to adapt the solution of the multistage stochastic problem to the actual PV generation and load request.

\section{A. Generation of scenarios}

In general, the number of scenarios for adequately describing this kind of stochastic process should be appropriately large. For the test 200 scenarios are generated to limit the computational time required by the Monte Carlo method.

For the scenario generation, we have applied the procedure described in e.g. [10], which includes a Markov-process to represent the autocorrelation that exists between consecutive observations. Starting from the forecasted profiles $P_{t}^{p v}$ and $P_{t}^{\text {load }}$, at first they are normalized by using the corresponding mean value and standard deviation; then, for each scenario $\omega$, the normalized time series $y_{t}^{p v}$ and $y_{t}^{\text {load }}$ are given by

$$
\begin{aligned}
& z_{\omega, t}=x_{\omega, t}+y_{t} \\
& x_{\omega, t}=\phi \cdot x_{\omega, t-1}+\varepsilon_{\omega, t}
\end{aligned}
$$

where $\phi$ is the one-lag autocorrelation parameter, assumed to be equal to 0.999 , and $\varepsilon_{\omega, t}$ is a Gaussian white noise with mean zero and standard deviation $\sqrt{1-\phi^{2}}$. The PV production and load profiles for each scenario $\omega\left(P_{\omega, t}^{p v}\right.$ and $\left.P_{\omega, t}^{\text {load }}\right)$ are obtained by applying the inverse transform method assuming a normal distribution, with the constraint that both profiles cannot be negative and that the difference between each profile and the corresponding forecast should not exceed $20 \%$ (in all the periods for the load and $75 \%$ of the periods for $\mathrm{PV}$ production).

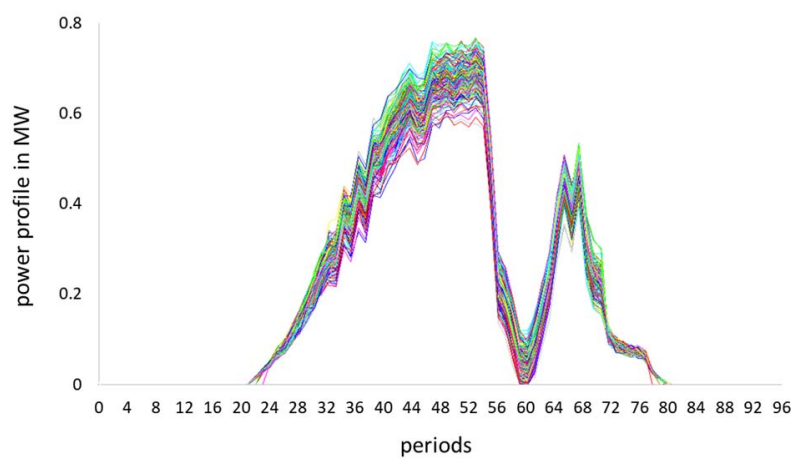

a)

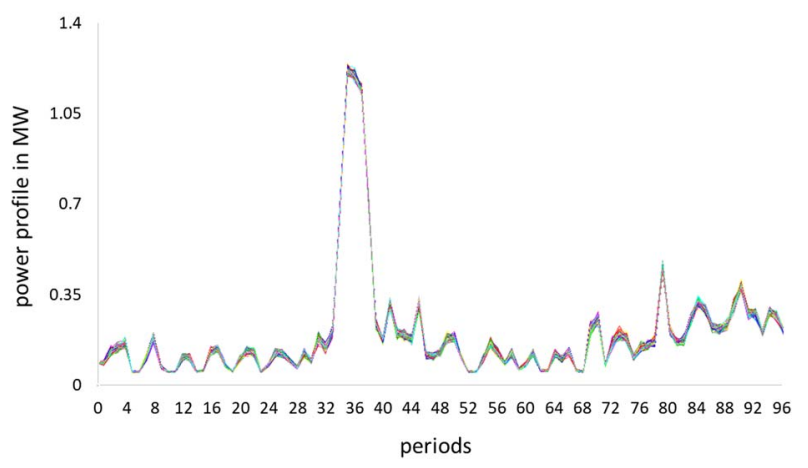

Fig. 2 Scenarios: a) PV production; b) load.

\section{B. Construction of the scenario tree}

Each of the generated scenarios is assumed to be equiprobable and it is defined by the normalized difference between the PV production and the load:

$$
\xi_{\omega, t}=\frac{P_{\omega, t}^{p v}-P_{\omega, t}^{\text {load }}}{P_{t}^{p v}-P_{t}^{\text {load }}}
$$

The scenario tree is built by the consecutive application of the $k$-means clustering method, as described in e.g. [11]. The main steps of this method are the following. 
At stage $s=1$ (that includes only period $t=0$ ), all scenarios have the same value of parameter, i.e. $\xi_{\omega, t=0}=\xi_{0}$

At stage $s=2(t=1 \ldots 24)$, the set of individual scenarios is divided in the predefined number $K$ of desired clusters $C_{s}^{k}$ (in the numerical tests, we compare the results obtained by using $K=3$ or $K=4$ ). At first, the initial $K$ centers $\bar{\xi}_{t}{ }^{k}$ are randomly selected; then each scenario $\xi_{\omega, t}$ is assigned to cluster $C_{s}^{k}$ so to minimize the dissimilarity measure

$$
d\left(\xi_{\omega, t}, \bar{\xi}_{t}^{k}\right)=\sum_{t \in T_{s}}\left\|\xi_{\omega, t}-\bar{\xi}_{t}^{k}\right\|_{2} \quad \forall k=1 \ldots K
$$

where \|\|$_{2}$ indicate the Euclidean distance and $T_{s}$ is the set of periods in stage $s$. After, the center of each cluster is updated as the mean of all the scenarios assigned to the cluster and the procedure is repeated until the centers of the clusters are not modified in two consecutive iterations.

The probability of each cluster at the considered stage $\pi_{s}^{k}$ is the sum of the probabilities of the individual scenarios belonging to the cluster. All the scenarios of the same cluster are replaced by the relevant center, i.e. $\xi_{\omega, t}=\bar{\xi}_{t}^{k} \quad \forall t \in T_{s}$ if $\xi_{\omega, t} \in C_{s}^{k}$.

At the stages following the second one, the $k$-means clustering algorithm is applied independently to each cluster defined in the previous stage.

The above-described procedure generates the scenario tree consisting, at each stage $s$, of nodes $\bar{\xi}_{t \in T_{s}}^{k}$ with the associated probabilities and the branches that connect nodes at different stages. The scenario tree used in the numerical tests for $K=3$ is shown in Fig.3.

The solution of the recourse model provides the optimal value of the decision variable in each node of the scenario tree at stage 1 (i.e. at $t=0$ ) and the beginning of each of the following stages from 2 to 5 (i.e., $t=24, t=48$, and $t=72$ ). The values of all other variables are calculated also at the end of each stage.

\section{Intraday decision-making procedure}

The solution of the recourse model provides multiple possible decisions at each stage following the first one (i.e., during the day). Therefore, for the actual operation, a decision-making procedure is needed for the choice of the most appropriate decision at each stage among those indicated by the stochastic problem solution, on the basis of the current PV generation and load.

At stage $s=2$, the decision-making procedure finds the scenario of the tree that is the most similar to the profile of the difference between PV generation and load in the previous 6 hours, on the basis of the Euclidean distance. Then it decides the set point values of the battery power output for each 15-minutes time intervals of the following 6-hours.

At stages $s=3$ and $s=4$, the decision-making procedure finds the scenario of the tree that is the most similar to the profile of the difference between PV generation and load in the previous
6 hours, only among those directly connected to the node chosen in the previous stage.

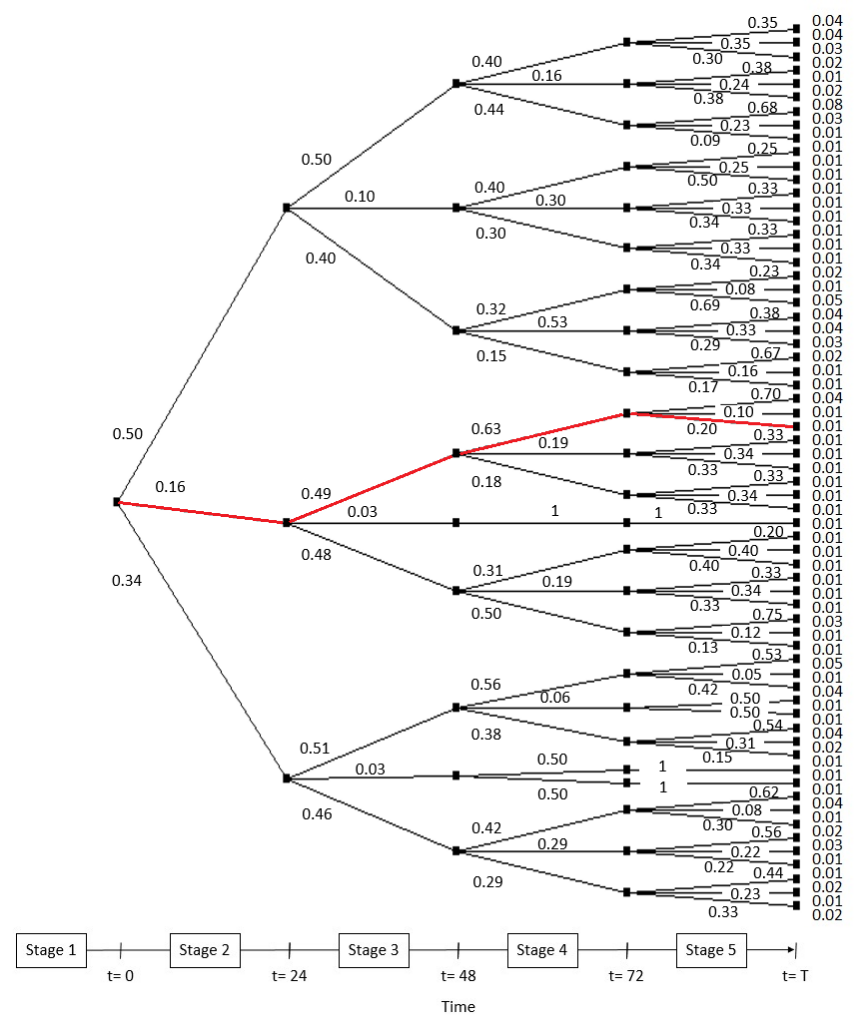

Fig. 3 Scenario tree obtained for 200 initial scenarios and 3 centroids. In red, an example of the solution provided by the decision-making function.

\section{NUMERICAL TESTS}

The optimization procedures have been implemented in AIMMS Developer and tested by using the Cplex V12.8 MIP solver on 2-GHz processors with $8 \mathrm{~GB}$ of RAM, running 64-b Windows.

Table I compares the $O F$ values of the stochastic solution of the two models (the one simple battery representation and the one including the KiBaM) by using the scenarios trees obtained through the $k$-means clustering procedure (with 3 and 4 centroids) applied to 200 initial equiprobable PV generation and load profiles.

TAB. I SP SOLUTIONS AND METRICS FOR THE CASE WITH A $630 \mathrm{kWh}$ BATTERY.

\begin{tabular}{|c|c|c|c|c|}
\hline Battery model & \multicolumn{2}{|c|}{ Simple } & \multicolumn{2}{c|}{ KiBaM } \\
\hline Number of centroids & 3 & 4 & 3 & 4 \\
\hline $\boldsymbol{O F}(\boldsymbol{\epsilon})$ & 38.02 & 38.25 & 61.67 & 61.80 \\
\hline $\boldsymbol{V S S}(\boldsymbol{\epsilon})$ & 2.59 & 2.84 & 1.11 & 1.12 \\
\hline $\boldsymbol{E V P I}(\boldsymbol{\epsilon})$ & 0.85 & 1.08 & 0.47 & 0.56 \\
\hline Number of scenarios in the tree & 64 & 139 & 64 & 139 \\
\hline Solution time (s) & 1.54 & 2.97 & 3.47 & 8.26 \\
\hline
\end{tabular}

Table I also shows the Value of Stochastic Solution (VSS) and the Expected Value of Perfect Information (EVPI), which are widely used metrics of the performance of using SP models [12].

VSS is the difference between the expected value solution 
(EEV) and the stochastic solution (i.e., the $O F$ value). $E E V$ is obtained by a two-step calculation: at first, the values of $P_{t}^{b}$ for each $t$ are given by the solution of the deterministic model obtained by replacing all random variables by their expected values; then, these $P_{t}^{b}$ are set as a fixed parameters and $E E V$ is given by the solution of the stochastic problem.

EVPI is the difference between the stochastic solution and the wait and see (WS) solution. WS is the expected value of the deterministic solutions of each scenario in the tree.

As expected, the higher the number of centroids the longer the computational effort due to the enlargement of the tree, as shown by the comparison of the solution times and the number of scenarios in the trees reported by Table I for $K=3$ and $K=4$. However, a more detailed clustering increases the VSS, even with an initial set of scenarios not very large with respect to the final dimensions of the tree.

Table I shows that the use of the more refined model of the battery increases both the $O F$ values and the computation time, as expected.

We have performed the same calculations also for a smaller battery $(315 \mathrm{kWh}$ instead of $630 \mathrm{kWh})$. The results are summarized in Table II and, they show a significant increase of $O F$ values, since the battery effect is less noticeable, and a decrease of both VSS and EVPI.

TAB. II SP SOLUTIONS AND METRICS FOR THE CASE WITH A $315 \mathrm{kWh}$ BATTERY.

\begin{tabular}{|c|c|c|c|c|}
\hline Battery model & \multicolumn{2}{|c|}{ Simple } & \multicolumn{2}{c|}{ KiBaM } \\
\hline Number of centroids & 3 & 4 & 3 & 4 \\
\hline $\boldsymbol{O F}(\boldsymbol{\epsilon})$ & 65.97 & 66.13 & 80.69 & 80.80 \\
\hline $\boldsymbol{V S S}(\boldsymbol{\epsilon})$ & 1.95 & 2.09 & 0.98 & 0.98 \\
\hline $\boldsymbol{E V P I}(\boldsymbol{\epsilon})$ & 0.48 & 0.63 & 0.43 & 0.52 \\
\hline Solution time (s) & 1.17 & 3.47 & 2.97 & 7.39 \\
\hline
\end{tabular}

As mentioned, this paper compares the SP approach with the Monte Carlo simulation technique, in which the deterministic model is solved for each initial scenario and then the $P_{t}^{b}$ values are set equal to the average of the corresponding values obtained by the deterministic solutions.

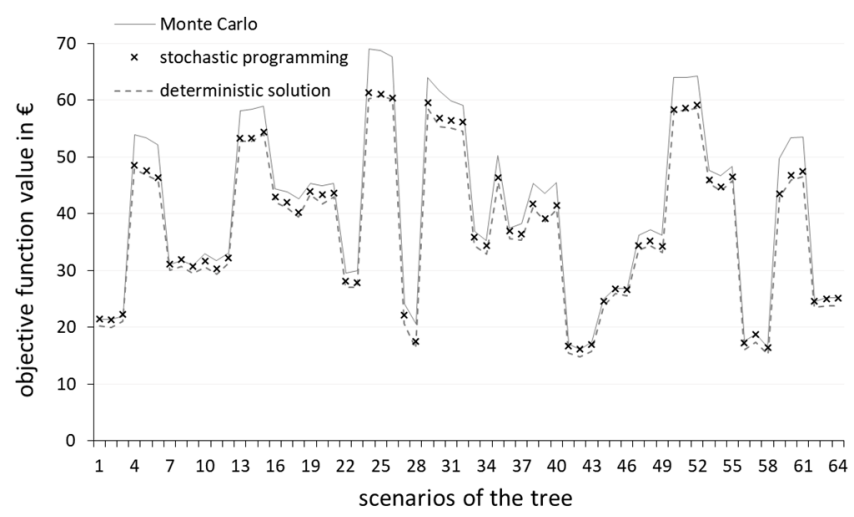

Fig. 4 Comparison between the values of the objective function for each scenario of the tree obtained by applying the $k$-means clustering with 3 centroids ( $630 \mathrm{kWh}$ battery).
Fig. 4 shows, for each scenario in the tree obtained with 3 centroids, the comparison between the $O F$ values calculated by using the Monte Carlo decisions and those given by the intraday decision-making procedure based on the SP solution. The figure also includes the $O F$ values of the deterministic solutions. SP provides in general better results with respect to Monte Carlo and this is confirmed also by Fig. 5 that shows the same comparison for 50 scenarios different from those included in the initial set. For the case of the small tree obtained by using 3 centroids the adoption of the SP approach needs also a shorter solution time, since the Monte Carlo simulations require around $5 \mathrm{~s}$ for the case of the simple battery model and $18 \mathrm{~s}$ if $\mathrm{KiBaM}$ is adopted, without parallel computing.

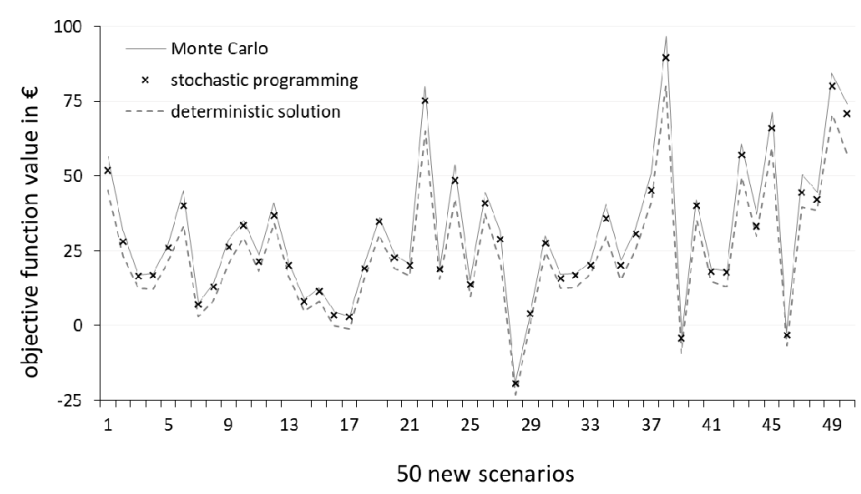

Fig. 5 Comparison between the values of the objective function for 50 new scenarios (630 kWh battery).

Table III shows the average values of the following differences for the scenarios of the tree, for the initial set of 200 scenarios, and for 50 scenarios different from those of the previous set:

SP-MC difference between the $O F$ values given by the intraday decision-making procedure and the Monte Carlo solution;

SP-WS difference between the $O F$ values given by the intraday decision-making procedure and the deterministic solution.

TAB. III. COMPARISON BETWEEN SP AND MONTE CARLO SIMULATIONS AND BETWEEN SP AND DETERMINISTIC SOLUTIONS (630 kWh BATTERY).

\begin{tabular}{|c|c|c|c|c|c|}
\hline \multicolumn{2}{|c|}{ Battery model } & \multicolumn{2}{c|}{ Simple } & \multicolumn{2}{c|}{ KiBaM } \\
\hline \multicolumn{2}{|c|}{ Number of centroids } & 3 & 4 & 3 & 4 \\
\hline \multirow{2}{*}{ Scenarios tree } & $\mathbf{S P}-\mathbf{M C}$ & -2.51 & -2.55 & -0.71 & -0.69 \\
\cline { 2 - 6 } & $\mathbf{S P}-\mathbf{W S}$ & 0.97 & 1.13 & 0.55 & 0.60 \\
\hline \multirow{2}{*}{$\begin{array}{c}\text { Set of initial } \\
\text { scenarios }\end{array}$} & $\mathbf{S P}-\mathbf{M C}$ & -2.05 & -2.47 & -0.23 & -0.34 \\
\cline { 2 - 6 } & $\mathbf{S P}-\mathbf{W S}$ & 5.17 & 2.70 & 1.81 & 1.70 \\
\hline \multirow{2}{*}{$\begin{array}{c}\text { Set of new } \\
\text { scenarios }\end{array}$} & $\mathbf{S P}-\mathbf{M C}$ & -2.28 & -2.29 & -0.26 & -0.24 \\
\cline { 2 - 6 } & $\mathbf{S P}-\mathbf{W S}$ & 4.85 & 4.84 & 1.91 & 1.92 \\
\hline
\end{tabular}

The results of Table III show the advantage of using the SP and the benefit of a more accurate clustering procedure. We have repeated the comparisons also for the case of the 315 $\mathrm{kWh}$ battery and the results, shown in Table IV, confirm in general the advantages of using 4 centroids although the average differences are smaller than in Table III. 
TAB. IV. PERFORMANCE COMPARISON FOR THE BATTERY OF $315 \mathrm{KWH}$

\begin{tabular}{|c|c|c|c|c|c|}
\hline \multicolumn{2}{|c|}{ Battery Model } & \multicolumn{2}{c|}{ Simple } & \multicolumn{2}{c|}{ KiBaM } \\
\hline \multicolumn{2}{|c|}{ Number of Centroids } & 3 & 4 & 3 & 4 \\
\hline \multirow{2}{*}{ Scenarios Tree } & SP - MC & -1.61 & -1.58 & -0.60 & -1.58 \\
\cline { 2 - 6 } & SP - WS & 0.56 & 0.66 & 0.49 & 0.55 \\
\hline \multirow{2}{*}{$\begin{array}{c}\text { Set of initial } \\
\text { Scenarios }\end{array}$} & SP - MC & -1.13 & -1.44 & -0.26 & -0.35 \\
\cline { 2 - 6 } & SP - WS & 2.21 & 1.91 & 1.60 & 1.50 \\
\hline $\begin{array}{c}\text { Set of New } \\
\text { Scenarios }\end{array}$ & $\mathbf{S P}-\mathbf{M C}$ & -1.12 & -1.14 & -0.30 & -1.14 \\
\cline { 2 - 6 } & $\mathbf{S P}-\mathbf{W S}$ & 2.81 & 2.79 & 1.67 & 2.79 \\
\hline
\end{tabular}

Finally, in order to show the performance of the SP approach under different conditions, the results obtained by using a new set of load scenarios of Fig. 6 (that replace those of Fig. 2b) are summarized in Table V ( $O F$ values and metrics) and Table VI (comparison between SP and Monte Carlo simulations and between SP and deterministic solutions over several sets of scenarios).

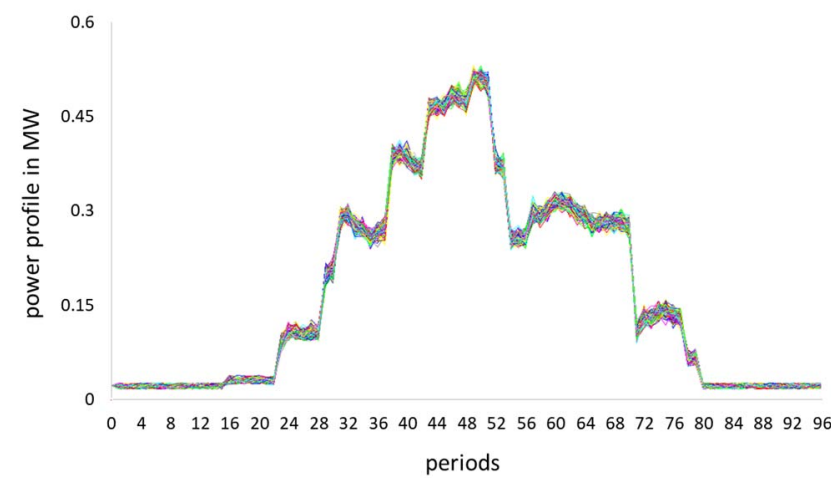

Fig. 6. Scenarios obtained for a different load profile forecast.

TAB. V. SP SOLUTIONS AND METRICS FOR THE CASE WITH A $630 \mathrm{kWh}$ BATTERY AND LOAD SCENARIOS OF FIG. 6 .

\begin{tabular}{|c|c|c|c|c|}
\hline Battery Model & \multicolumn{2}{|c|}{ Simple } & \multicolumn{2}{c|}{ KiBaM } \\
\hline Number of Centroids & 3 & 4 & 3 & 4 \\
\hline $\boldsymbol{O F}(\boldsymbol{\epsilon})$ & -2.94 & -2.33 & 9.54 & 9.84 \\
\hline $\boldsymbol{V S \boldsymbol { S } ( \boldsymbol { \epsilon } )}$ & 1.75 & 2.52 & 1.93 & 2.66 \\
\hline $\boldsymbol{E V P I}(\boldsymbol{\epsilon})$ & 2.66 & 3.19 & 0.96 & 1.19 \\
\hline Number of scenarios in the tree & 74 & 169 & 74 & 169 \\
\hline Solution time (s) & 1.53 & 4.45 & 3.91 & 8.87 \\
\hline
\end{tabular}

TAB. VI. COMPARISON BETWEEN SP AND MONTE CARLO SIMULATIONS AND BETWEEN SP AND DETERMINISTIC SOLUTIONS (630 KWH BATTERY AND LOAD SCENARIOS OF FIG. 6).

\begin{tabular}{|c|c|c|c|c|c|}
\hline \multicolumn{2}{|c|}{ Battery Model } & \multicolumn{2}{c|}{ Simple } & \multicolumn{2}{c|}{ KiBaM } \\
\hline \multicolumn{2}{|c|}{ Number of Centroids } & 3 & 4 & 3 & 4 \\
\hline \multirow{2}{*}{ Scenarios Tree } & SP - MC & -1.37 & -1.84 & -1.33 & -1.94 \\
\cline { 2 - 6 } & $\mathbf{S P}-\mathbf{W S}$ & 3.71 & 3.37 & 1.29 & 1.14 \\
\hline \multirow{2}{*}{$\begin{array}{c}\text { Set of initial } \\
\text { Scenarios }\end{array}$} & $\mathbf{S P}-\mathbf{M C}$ & -0.28 & -1.06 & -0.84 & -1.40 \\
\cline { 2 - 6 } & $\mathbf{S P}-\mathbf{W S}$ & 7.19 & 6.41 & 4.45 & 3.89 \\
\hline $\begin{array}{c}\text { Set of New } \\
\text { Scenarios }\end{array}$ & $\mathbf{S P}-\mathbf{M C}$ & 0.64 & -0.39 & -0.83 & -1.30 \\
\cline { 2 - 6 } & $\mathbf{S P}-\mathbf{W S}$ & 9.35 & 8.33 & 4.50 & 4.03 \\
\hline
\end{tabular}

The results confirm the advantages of the SP. Only in the case of the 50 new scenarios and the simple battery model using 3 Centroids, the average performance for the stochastic solution is higher than the one obtained by using the Monte Carlo solution. The use of 4 centroids increases the VSS and allows to reach improved results with respect to the Monte Carlo technique.

\section{CONCLUSION}

Multistage SP represents an attractive method for the day ahead scheduling in local energy systems and provides improved results with respect to the application of the Monte Carlo method.

The construction of the scenario tree needs to be addressed properly. The $k$-means clustering provides appropriate results even with a limited number of centroids. The computational effort is reasonable for the considered five-stage SP problem.

The SP approach is also applicable to models that include a detailed representation of the battery under the assumption that the mixed integer linear programming characteristics of the model are preserved.

\section{REFERENCES}

[1] S. S. Reddy, V. Sandeep, and C.-M. Jung, "Review of stochastic optimization methods for smart grid," Front. Energy, vol. 11, no. 2, pp. 197-209, 2017.

[2] G. C. Lazaroiu, V. Dumbrava, G. Balaban, M. Longo, and D. Zaninelli, "Stochastic optimization of microgrids with renewable and storage energy systems," EEEIC 2016 - Int. Conf. Environ. Electr. Eng., 2016.

[3] Y. Yuan, Q. Li, and W. Wang, "Optimal operation strategy of energy storage unit in wind power integration based on stochastic programming," IET Renew. Power Gener., vol. 5, no. 2, p. 194, 2011.

[4] J. F. Manwell and J. G. McGowan, "Lead acid battery storage model for hybrid energy systems," Sol. Energy, vol. 50, no. 5, pp. 399-405, May 1993.

[5] N. Daniil, D. Drury, and P. H. Mellor, "Performance comparison of diffusion, circuit-based and kinetic battery models," 2015 IEEE Energy Convers. Congr. Expo. ECCE 2015, pp. 1382-1389, 2015.

[6] C. Bordin, H. O. Anuta, A. Crossland, I. L. Gutierrez, C. J. Dent, and D. Vigo, "A linear programming approach for battery degradation analysis and optimization in offgrid power systems with solar energy integration," Renew. Energy, vol. 101, pp. 417-430, 2017.

[7] A. Sakti, K. G. Gallagher, N. Sepulveda, C. Uckun, C. Vergara, F. J. de Sisternes, D. W. Dees, and A. Botterud, "Enhanced representations of lithium-ion batteries in power systems models and their effect on the valuation of energy arbitrage applications," J. Power Sources, vol. 342, pp. 279-291, 2017.

[8] F. Conte, S. Massucco, M. Saviozzi, and F. Silvestro, "A Stochastic Optimization Method for Planning and Real-Time Control of Integrated PV-Storage Systems: Design and Experimental Validation," IEEE Trans. Sustain. Energy, vol. 3029, no. LV, pp. 1-10, 2017.

[9] S. Lilla, A. Borghetti, F. Napolitano, F. Tossani, D. Pavanello, D. Gabioud, Y. Maret, and C. A. Nucci, "Mixed integer programming model for the operation of an experimental low-voltage network," in 2017 IEEE Manchester PowerTech, 2017, pp. 1-6.

[10] G. J. Osório, J. M. Lujano-Rojas, J. C. O. Matias, and J. P. S. Catalão, “A new scenario generation-based method to solve the unit commitment problem with high penetration of renewable energies," Int. J. Electr. Power Energy Syst., vol. 64, pp. 1063-1072, 2015.

[11] H. Pranevicius and K. Sutiene, "Scenario tree generation by clustering the simulated data paths," in Proceedings 21st European Conference on Modelling and Simulation ECMS 2007, 2007, pp. 203-208.

[12] L. F. Escudero, A. Garín, M. Merino, and G. Pérez, "The value of the stochastic solution in multistage problems," Top, vol. 15 , no. 1 , pp. 48 $64,2007$. 